\title{
ODONTOMETRIC AND MORPHOLOGIC DISCREPANCY STUDY OF PRIMARY TEETH IN YEMENIS CHILDREN "A CROSS SECTIONAL STUDY"
}

\author{
Hani Al-Akhali *, Kamal El Din M. El- Mutayam ** \\ and Adel A. El-Bardissy ****
}

\begin{abstract}
The aim of the study was To collect normative data of crown size of primary dentition in a group of Yemenis Children and Compare the results we were found to previous published data of other Arab populations.
\end{abstract}

Subjects and methods: A total number of two hundred Yemeni children were included in this study, 100 males and 100 females, their ages ranged from 3-7 years old. Upper and lower impressions were taken. Each impression was poured immediately in stone plaster. A full set of primary teeth (incisors, canines, first and second molars) on both sides of the maxillary and mandibular dental arches were measured in Mesiodistal (MD) dimension.

Results: No statistically significant difference in the mean mesiodistal tooth width between the mesiodistal crown size obtained in the present study with those reported by other authors who studied other Arab populations.

Conclusions: The present values were numerically greater than, but close to the values obtained in Egyptian children, where all deciduous teeth were also evaluated. In Jordanian population that revealed largest mesiodistal value in comparison to the Egyptian and our study of Yemenis children.

KEY WORDS: Odontometric - Mesiodistal - Primary Teeth -Yemenis Children

\footnotetext{
* MSc in Pediatric Dentistry, Ass. Lecturer of Pediatric Dentistry Faculty of Dentistry, Thamar University - Yemen.

** Professor of Pediatric Dentistry, Faculty of Oral and Dental Medicine, Cairo University.

*** Ass. Professor of Pediatric Dentistry, Faculty of Oral and Dental Medicine, Cairo University.
} 


\section{INTRODUCTION}

In order to improve the quality of dental care available, there is a great need for data on the mesiodistal crown dimensions of the individual teeth of Yemenis children. Most data available on crown size analysis are for Northern European descent and no data available for Yemen population, this created the idea of the research as a part of a project applied on different Yemeni cities to collect data regarding mesiodistal widths of the primary mandibular incisors and maxillary teeth in a group of Yemenis children. Tooth measurement provides valuable data for anthropology and dentistry. These data are useful for restoring the crown of deciduous teeth and understanding the occlusion of deciduous dentition in pediatric dentistry ${ }^{1}$.

\section{SUBJECTS AND METHODS}

This cross-sectional study was conducted in Sana'a City, the Capital of Republic of Yemen; twenty one public primary schools were selected from the city. A total number of two hundred Yemeni children were included in this study, 100 males and 100 females, their ages ranged from 3-7 years old. Upper and lower impressions were taken. Each impression was poured immediately in stone plaster. A full set of primary teeth (incisors, canines, first and second molars) on both sides of the maxillary and mandibular dental arches were measured in Mesiodistal (MD) dimension. To ensure correct measurements, a special device was designed with a platform on which the cast to be placed and measurements were done directly on the plaster models with the digital caliper, which sharpened its tip to facilitate the insertion to the contact point, enabling to obtain values with accuracy of up to $0.01 \mathrm{~mm}$.

\section{RESULTS}

The results of this study revealed

- No statistically significant difference in the mean mesiodistal tooth width between the mesiodistal crown size obtained in the present study with those reported by ${ }^{2}$ in Egyptian children, where all deciduous teeth were also evaluated and Jordanian population ${ }^{3}$.

- When comparing the mesiodistal crown size obtained in the present study with those reported by other authors, no specific pattern was observed. The present values were numerically greater than, but close to the values obtained by ${ }^{(2)}$ in Egyptian children, where all deciduous teeth were also evaluated.

- The study of Jordanian population ${ }^{3}$ that revealed largest mesiodistal value in comparison to the Egyptian and our study of Yemeni children.

\section{DISCUSSION}

In this study the mesiodistal tooth width of mandibular and maxillary primary teeth in a sample of two hundred children with an age range from three to sevenyears were studied. This range of age was selected so that one could study the primary dentition with no or minimum wear on the proximal surface. Measurements were taken with the aid of a digital caliper and study casts for each child. A special device was designed with a platform on which the cast to be placed and fixed with screws that facilitate the standardization of measurements. The procedures for measuring the mesiodistal tooth width were performed as described by ${ }^{4}$ as the greatest mesiodistal measurement from the anatomic mesial contact point to the anatomic distal contact point of each tooth, measured parallel to the occlusal plane.Digital caliper (Mitutoyo, Japan) 
Accuracy $(0.01 \mathrm{~mm})$ were advocated for scientific use and this was in harmony with ${ }^{5}$ who showed that digital calipers were more accurate when used to measure mesiodistal tooth width on study casts as compared to the measurements taken on virtual computerized models (OrthoCad).

\section{CONCLUSIONS}

The comparing between the published data of Arab population and the result of our present study:

- The Egyptian and Jordanian population, When comparing the mesiodistal crown size obtained in the present study with those reported by other authors, no specific pattern was observed. The present values were numerically greater than, but close to the values obtained by ${ }^{(2)}$ in Egyptian children, where all deciduous teeth were also evaluated.
- The study of Jordanian population ${ }^{(3)}$ that revealed largest mesiodistal value in comparison to the Egyptian and our study of Yemeni children.

\section{REFERENCES}

1- Tsai HH.: Dental crowding in primary dentition and its relationship to arch and crown dimensions. J Dent Child. 2003;70(2):164-9.

2- El-Nofely, L. Sadek And N. Soliman : Spacing in the human deciduous dentition inrelation to tooth size and dental arch size. Archs oral Biol. Vol. 34, No. 6, pp. 437441, 1989

3- Hattab FN, al-Khateeb S, Sultan I.: Mesiodistal crown diameters of permanent teeth in Jordanians. Arch Oral Biol. 1997; 41(7):641-5.

4- Hunter, S.W. and Priest, R.W.: Errors and discrepancies in measurement of tooth size. J. Dent. Res. 39:405-414, 1960.

5- Zilberman L.R.: Tooth measurments on virtual computerized models (OrthoCad) and digital calipers. Am J Orthod 79:343-37, 2003. 